\title{
A Case Report about Persistent Left Superior Vena Cava: Is it Always Asymptomatic?
}

\author{
Özge Çetinarslan, Arda Payas, Ibrahim Taskin Rakici
}

Private Incirli Ethica Hospital, Istanbul, Turkey

Email: ozgecetinarslan@windowslive.com

How to cite this paper: Çetinarslan, Ö., Payas, A. and Rakici, I.T. (2021) A Case Report about Persistent Left Superior Vena Cava: Is it Always Asymptomatic? World Journal of Cardiovascular Diseases, 11, 52-57. https://doi.org/10.4236/wjcd.2021.111007

Received: November 30, 2020

Accepted: January 23, 2021

Published: January 26, 2021

Copyright $\odot 2021$ by author(s) and Scientific Research Publishing Inc. This work is licensed under the Creative Commons Attribution International License (CC BY 4.0).

http://creativecommons.org/licenses/by/4.0/

(c) (i) Open Access

\begin{abstract}
A persistent left superior vena cava (PLSVC) is a rare malformation which affects approximately $0.3 \%-0.5 \%$ of the population and it is presented along with a right-sided superior vena cava in $82.2 \%$ of the cases reported [1]. Clinicians diagnose it incidentally by difficulties with pacemaker implantation, central venous catheterization or screening for another etiologies when it is not accompanied by other anomalies it is typically asymptomatic. W. Schummer et al. described the embryogenesis and the anatomic variations of persistent LSVC according to the positioning of a central venous catheter on the chest radiograph: type I, normal; type II, only PLSVC; type IIIa, right and left superior vena cava with connection; type IIIb, right and left superior vena cava without connection [2]. In 92\% of individuals with PLSVC, the PLSVC drains into a dilated coronary sinus (CS) and rest $8 \%$ drain directly into the left atrium. PLSVC is caused by a failure in the closure of the left anterior cardinal vein during embryogenic development [3]. The coronary sinus (CS) is a vein that transmits venous blood to the right atrium though atrioventricular groove. The CS wall contains atrial myocardium. Thus, its size extensively depends on variability of blood flow and pressure. We present a variant PLSVC with unknown prevalence and a mild platypnea-orthodeoxia syndrome after recovery of COVID-19 related acute respiratory distress syndrome (ARDS).
\end{abstract}

\section{Keywords}

Case Report, Persistent Left Superior Vena Cava (PLSVC), Congenital Heart Disease, COVID-19, Cardiac Imaging

\section{Introduction}

In this case report, we present a 51 years old male who cannot be discharged from hospital due to somewhat platypnea and orthodeoxia-like syndromes after recovery of COVID-19. The patient was intubated for 20 days and received con- 
valescent plasma therapy along with his medical treatment for COVID-19 related ARDS (favipiravir for 5 days po, sc LMWH $6000 \mathrm{IU} / 0.6 \mathrm{ml}$ twice a day, acetylsalicylic acid $100 \mathrm{mg}$ once a day po, iv methylprednisolone $40 \mathrm{mg}$ twice a day, iv paracetamol $10 \mathrm{mg} / \mathrm{ml}$ twice a day, iv piperacillin tazobactam $4.5 \mathrm{~g}$ three times a day and moxifloxacin once a day for ten days). His medical history was clear completely, his body mass index was 24 and he had never smoked. His unexpected hypotension $(80 / 50 \mathrm{mmHg})$ and mild desaturation (70\%) remained even after giving enough time for orthostasis and over hydration for three days. His inflammation markers were in normal range. COVID-19 PCR test was negative for last two times. Thorax computer tomography (CT) revealed mild signs of past ARDS and there was not any pulmonary embolism. His electrocardiogram and other biochemical values were insignificant. Finally, we discovered coronary sinus dilatation $(31 \times 24 \mathrm{~mm})$ by transthoracic echocardiography (TTE) examination with normal size heart chambers and non-pathological heart valves. The left ventricular ejection fraction was $60 \%$ and the estimated peak systolic pulmonary artery pressure was $25 \mathrm{mmHg}$ (Figure 1). A contrast echocardiogram with agitated saline administration through the left antecubital vein showed air bubbles in coronary sinus before right atrium. In addition, no interatrial shunt was revealed spontaneously or with Valsalva maneuver. This vision suggested PLSVC and we asked for a new CT interpretation to radiology.

MDCT revealed a bridging vein draining the right jugular and right subclavian veins; it formed the PLSVC, which descended along the right side of thoracic vertebra, leftward of the pulmonary artery and left atrium (LA) before partial draining into the right atrium (RA) via a dilated CS (Figure 2, Figure 3). Then, left-sided paramediastinal SVC descended into the hemiazygos vein with collaterals crossing midline from left to right into the azygos vein. The azygos vein, in turn, drained directly into the right SVC (Figure 4, Figure 5).

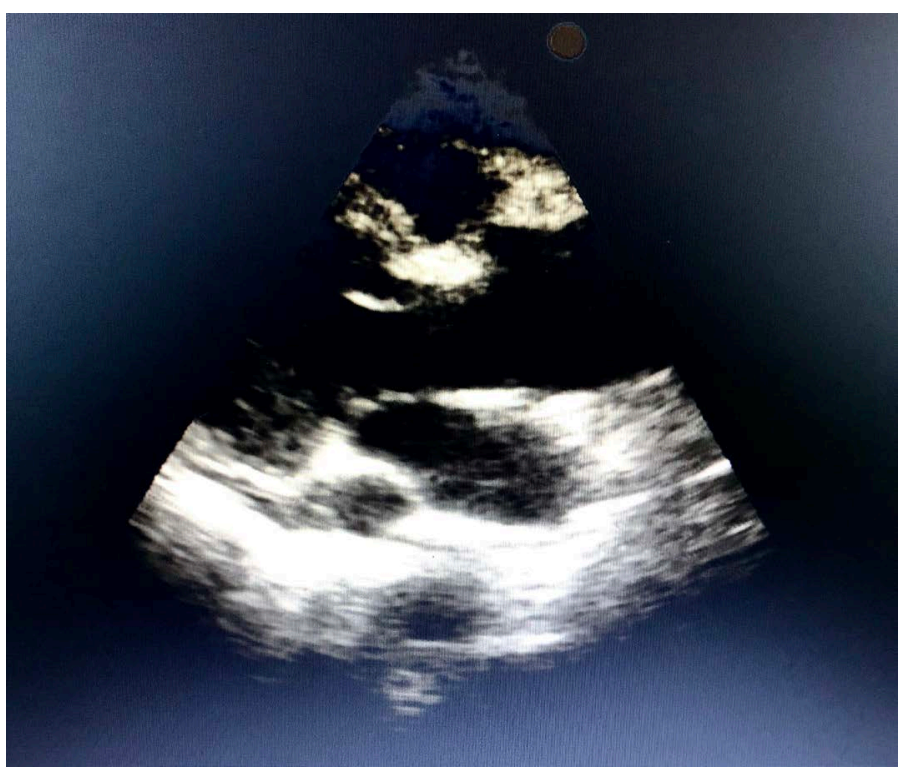

Figure 1. Enlargement of coronary sinus on transthoracic echocardiography. 


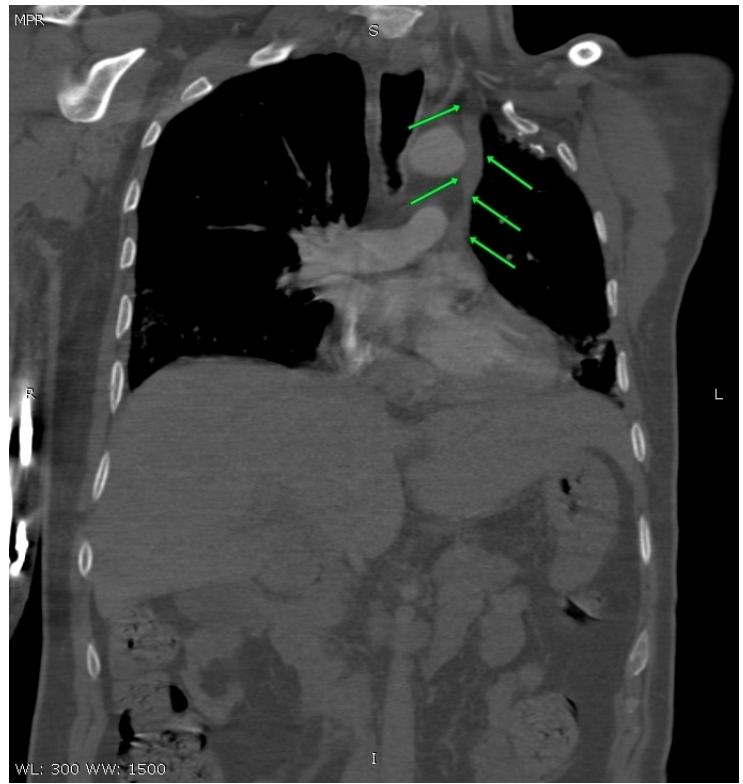

Figure 2. Coronal multiplanar reformatted CT pulmonary angiography image reveals the persistent left superior vena cava (PLSVC) pointed by green arrows.

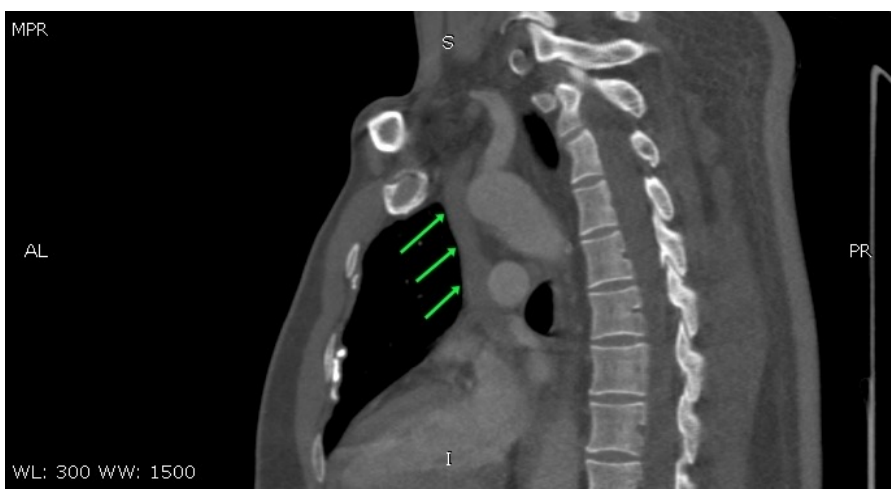

Figure 3. Sagittal multiplanar reformatted CT pulmonary angiography image reveals the persistent left superior vena cava (PLSVC) draining into a dilated coronary sinus (CS).

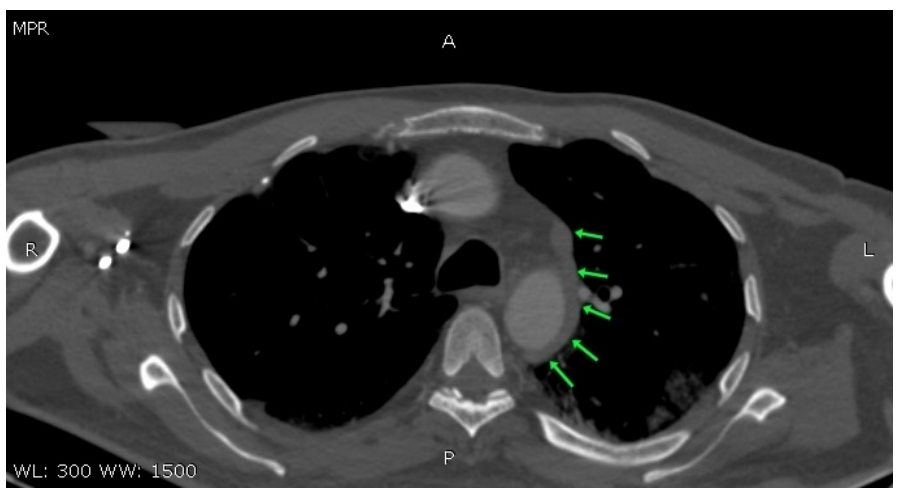

Figure 4. Sagittal multiplanar reformatted CT pulmonary angiography image demonstrates PLSVC descended along the right side of thoracic vertebra, leftward of the pulmonary artery and left atrium (LA) before partial draining into the right atrium (RA) via a dilated coronary sinus (CS). Then left-sided paramediastinal SVC descended into the hemiazygos vein with collaterals crossing midline from left to right into the azygos vein. 


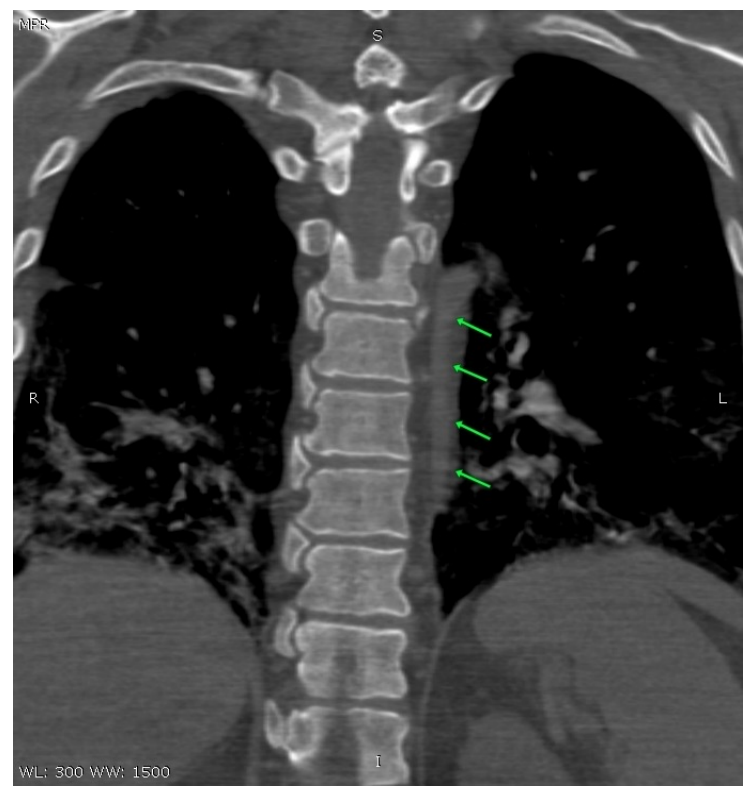

Figure 5. Axial reformatting of CT Pulmonary demonstrates PLSVC with continuation into the hemiazygos vein. Collaterals cross midline from left to right into the azygos vein to connect to the azygos vein on the right side with subsequent drainage into the right superior vena cava (SVC).

PLSVC related cardiac anomalies such as atrial septal defect, endocardial cushion defects, tetralogy of Fallot, coarctation of aorta, bicuspid aortic valve and partial or complete venous return anomaly were not detected by CT. We just continued to parenteral hydration and mobilization tries to allow accommodation. The diameter of CS decreased from $3.0 \mathrm{~cm}$ to $2.0 \mathrm{~cm}$ before discharge after following for a week. His saturation was $>90 \%$ and blood pressure was normal. There was no headache or dizziness anymore. The patient's informed consent was obtained to report this case.

\section{Discussion}

During normal foetal development, failure of the regression of the left anterior cardinal vein results in PLSVC [4]. When failure of closure occurs at an earlier stage, the CS is absent and the PLSVC drains into the left atrium. Either isolated or associated with RSVC, this venous malformation itself is usually diagnosed incidentally [5] [6].

If PLSVC is not associated with other congenital cardiac abnormalities, it is usually hemodynamically insignificant. However, as in our case, lying back for a long time can cause a requirement for healthy venous return and become intolerant to any kind of venous return anomaly. Even, awareness about enlargement of CS and mechanism of LPSVC is important before some interventional and surgery procedures.

There are several reported cases of a PLSVC with a connection to the azygous system or right atria via coronary sinus [7]. In our patient, the PLSVC descended along the along the right side of thoracic vertebra, leftward of the pulmonary ar- 
tery and LA before draining into the right atrium (RA) via a dilated CS partially, and then crossed the midline to connect to the azygos vein on the right side. After forming a connection with the azygos vein, it followed its normal anatomical course back to the heart.

\section{Conclusion}

PLSVC is a rare congenital variation. If it is not associated with congenital heart disease, it is usually asymptomatic [8]. Our case is important due to the fact that our patient presented with platypnea and orthodeoxia after recovery of COVID-19 while all of the tests were normal. CT made the diagnosis of a PLSVC with a dilated CS and in the absence of a congenital heart disease. A CS enlargement should lead us to search for PLSVC even it is asymptomatic in stable condition and the clinicians should keep in mind that any hemodynamic instability can make PLSVC a significant clinical manifestation.

\section{Conflicts of Interest}

The authors declare no conflicts of interest regarding the publication of this paper.

\section{References}

[1] Danielpour, P.J., Aalberg, J.K., El-Ramey, M., Sivina, M. and Wodnicki, H. (2005) Persistent Left Superior Vena Cava: An Incidental Finding during Central Venous Catheterization-A Case Report. Vascular and Endovascular Surgery, 39, 109-111. https://doi.org/10.1177/153857440503900111

[2] Schummer, W., Schummer, C. and Fröber, R. (2003) Persistent Left Superior Vena Cava and Central Venous Catheter Position: Clinical Impact Illustrated by Four Cases. Surgical and Radiologic Anatomy, 25, 315-321. https://doi.org/10.1007/s00276-003-0138-6

[3] Burney, K., Young, H., Barnard, S.A., McCoubrie, P. and Darby, M. (2007) CT Appearances of Congential and Acquired Abnormalities of the Superior Vena Cava. Clinical Radiology, 62, 837-842. https://doi.org/10.1016/j.crad.2007.04.001

[4] Fischer, D.R. and Zuberbuhler, J.R. (2002) Paediatric Cardiology. In: Anderson, R.H., Baker, E. and Mccartney, R.F., Eds., Anomalous Systemic Venous Return, Churchill Livingstone, New York, 851-865.

[5] Shyamkumar, N.K. and Brown, R. (2007) Double Superior Vena Cava with a Persistent Left Superior Vena Cava: An Incidental Finding during Peripherally Inserted Central Catheter Placement. Australasian Radiology, 51, 257-259. https://doi.org/10.1111/j.1440-1673.2007.01796.x

[6] Antretter, H., Cottogni, M., Oberhauser, A., Lhotta, K. and Furtwängler, W. (1992) Pacemaker Implantation via a Persistent Left Superior Vena Cava in Atresia of the True Superior Vena Cava. Deutsche Medizinische Wochenschrift, 117, 1394-1398. https://doi.org/10.1055/s-2008-1062456

[7] Sakamoto, H., Akita, K., Sato, K. and Sato, T. (1993) Left Superior Vena Cava Continuing to the Accessory Hemiazygos without Anastomosis with the Coronary Sinus. Surgical and Radiologic Anatomy, 15, 151-154. https://doi.org/10.1007/BF01628317 
[8] Bolognesi, M. (2015) Dilated Coronary Sinus due to Persistent Left Superior Vena Cava in a Healthy Athlete: A Case Report with Brief Review. Journal of Integrative Cardiology, 1, 115-117. https://doi.org/10.15761/JIC.1000131 